\title{
Clinical and imaging interrelationships in the diagnosis of foreign body aspiration in children
}

\section{*Diana Rotaru-Cojocari, Victor Rascov, Rodica Selevestru, Svetlana Sciuca}

\author{
Department of Pediatrics, Nicolae Testemitanu State University of Medicine and Pharmacy
}

Chisinau, the Republic of Moldova

\author{
Authors' ORCID iDs, academic degrees and contributions are available at the end of the article \\ ${ }^{*}$ Corresponding author - Diana Rotaru-Cojocari, e-mail: dianarotaru2@gmail.com \\ Manuscript received October 18, 2021; revised manuscript November 16, 2021; published online November 26, 2021
}

\begin{abstract}
Background: Foreign body aspiration (FBA) is a typical occurrence in children. The clinical signs are influenced by various causes, and the differential diagnosis is important, especially when the suffocation crisis is not recognized. The aim of this study was to evaluate the clinical and imaging symptoms in children with FBA.

Material and methods: A retrospective study is provided of 156 children who were hospitalized and examined (clinical and paraclinical tests) in the Pneumology Clinic between 2011 and 2020 after having a foreign body removed from their airways, using rigid tube bronchoscopy or fibrobronchoscopy. Results: The most affected age group was 1-3 years, which constituted 77.6\% (95\% CI 70.2\% -83.8\%). The most common symptoms were: cough - 98.7\% (95\% CI 95.4\%-99.8\%), dyspnoea - 94.2\% (95\% CI 89.3\%-97.3\%), wheezing - 61.5\% (95\% CI 53.4\%-69.2\%). Chest radiography was relevant for foreign body aspirations in 55.8\% of cases (95\% CI 47.6\%-63.7\%). The foreign body was extracted from the right bronchus in $32.1 \%$, from the left bronchus in $21.8 \%$ of cases, from the lobar / segmental bronchi $-22.5 \%$, and in $21.2 \%$ - multiple locations. The etiological structure of the endobronchial foreign body was dominated by the organic ones $-96.8 \%$.

Conclusions: Cough, dyspnoea, and wheezing are suggestive of this pediatric emergency. Chest radiography provides diagnostic information only for every second child.

Key words: foreign body, children, bronchoscopy.
\end{abstract}

\section{Cite this article}

Rotaru-Cojocari D, Rascov V, Selevestru R, Sciuca S. Clinical and imaging interrelationships in the diagnosis of foreign body aspiration in children. Mold Med J. 2021;64(5):47-50. https://doi.org/10.52418/moldovan-med-j.64-5.21.09.

\section{Introduction}

Foreign body aspiration is a common pathology in the pediatric population, with various forms of presentation ranging from acute symptoms accompanied by life-threatening respiratory failure due to total airway obstruction to mild recurrent respiratory manifestations, with exacerbations that alternate with periods of apparent health [1]. It is critical in both situations to make a differential diagnosis of airway blockage, which might be caused by a foreign body aspiration, infections, or an allergic bronchopulmonary disease $[2,3]$.

The clinical picture of foreign body aspiration events in the airways is determined by several parameters, including the degree of installation in the respiratory system, the type and pathogenic mechanism of the blockage, the nature of the foreign body, and the time between diagnosis and treatment [4-7]. Fixation of the foreign body to the tracheal bifurcation results in abrupt death in the middle of severe suffocation or cardiorespiratory arrest. Furthermore, the progression of this pediatric emergency differs depending on whether the bronchi are partially or completely blocked, or if the valve mechanism is used. Organic foreign bodies create greater problems since they are non-contrasting to chest radiography most of the time, undergo putrefaction processes over time, and are difficult to remove using bronchoscopic methods. Sharp foreign bodies, particularly metal foreign bodies formed of hard plastic, can cause mechanical damage to the bronchial shaft, increasing the likelihood of developing catarrhal-purulent endobronchitis and granulations, and in some circumstances can breach the bronchial wall.

With a sensitivity of $96 \%$ and a specificity of $76 \%$, a suffocation episode is one of the most reliable clinical indicators of foreign body aspiration $[8,9]$. However, only a tiny number of patients exhibit the typical triad, which includes suffocation, coughing, and wheezing, and the penetration event frequently goes undetected or ignored, complicating the diagnosis of foreign body aspiration $[6,10,11]$. In the absence of particular therapy, such as bronchoscopy and foreign body extraction, it encourages the development of complications, some of which are followed by persistent broncho-pulmonary sequelae, bronchopulmonary processes with chronic characteristics [12-16]. 
Table 1. General and clinical characteristics of pediatric patients with foreign body aspiration

\begin{tabular}{|c|c|c|c|c|c|c|c|}
\hline Age group & No of cases & $\%$ & $95 \% \mathrm{Cl}$ & Gender & No of cases & $\%$ & $95 \% \mathrm{Cl}$ \\
\hline$<1$ year & 20 & 12.8 & $8.0-19.1$ & Male & 89 & 57.1 & $48.9-64.9$ \\
\hline $1-3$ years & 121 & 77.6 & $70.2-83.8$ & Female & 67 & 42.9 & $35.1-55.1$ \\
\hline$>3$ years & 15 & 9.6 & $5.5-15.4$ & & & & \\
\hline \multicolumn{8}{|c|}{ The main symptoms } \\
\hline Symptom & No of cases & $\%$ & $95 \% \mathrm{Cl}$ & Symptom & No of cases & $\%$ & $95 \% \mathrm{Cl}$ \\
\hline Stridor & 9 & 5.8 & $2.7-10.7$ & Cough & 154 & 98.7 & $95.4-99.8$ \\
\hline Fever & 64 & 41.0 & $33.2-49.2$ & Wheezing & 96 & 61.5 & $53.4-69.2$ \\
\hline Perioral cyanosis & 59 & 37.8 & $30.2-45.9$ & Dyspnoea & 147 & 94.2 & $89.3-97.3$ \\
\hline
\end{tabular}

\section{Material and methods}

The study group included 156 children hospitalized in the Pneumology Clinic of Institute of Mother and Child, in a period of 10 years (2011-2020). The investigation was conducted retrospectively and was based on the review of patient observation records. A total of 66 patients were targeted with suspicion for FBA, and the diagnosis was suspected and confirmed in 90 patients while they were in the hospital. Age, gender, the reason for hospitalization, time from aspiration to diagnosis, clinical symptoms, and radioimaging characteristics were all tracked for all patients. Endobronchial foreign body was diagnosed using rigid tube bronchoscopy (Karl Sorz 3.5-6.0mm) or fibrobronchoscopy (BF3C160, BF1TQ170 Olympus 3.8-6.0mm), depending on the case.

\section{Results}

The examination by age groups in the study group revealed a net prevalence of children aged 1-3 years - 121 cases $(77.6 \%)$, and babies in 20 cases $(12.8 \%)$. Aspiration of foreign bodies in the respiratory system had only a modest, statistically insignificant male predominance: 89 cases in boys (57.1\%) and 67 instances in girls (42.9\%) (tab. 1). In terms of location, $68.58 \%$ of patients (107 children) are from country areas, whereas $31.41 \%$ (49 patients) are from city areas.

There was a varying time delay for each case, varying from one hour to 6 months, between the time the foreign body was aspirated and the request for a medical consultation. Only $66.0 \%$ of cases ( 103 children) had the penetration syndrome recognized anamnestically, but only one-third of these patients presented to the hospital during the first three days of the aspiration incident. In these cases, the diagnosis was simple and quick to confirm, and the treatment entailed the removal of foreign bodies from the bronchial tree as soon as possible.

The most common symptoms seen in the clinical presentation of foreign body aspiration cases were: cough, which was present in 154 instances (98.7\%), dyspnoea of different severity verified in 147 children (94.2\%), and wheezing in 96 cases $(61.5 \%)$ (tab. 1). Bronchopulmonary physical examination revealed: decreased vesicular resonance in 75 cases $(48.07 \%)$, wet rales in 88 cases $(56.41 \%)$, wheezing rales in 111 cases $(71.15 \%)$, and no stetoacoustic changes in 4 cases $(2.56 \%)$.

The radiological examination was performed in all patients, but no radiopaque foreign materials were detected, and the radiography in 13 cases did not demonstrate patho- logical changes, with a normal image. In the majority of instances (55.8 \% (95\% CI 47.6\%-63.7\%)), indirect radiological signals were indicating the presence of a foreign body in the bronchial tree, such as atelectasis in $25 \%$ of cases, localized emphysema in $48 \%$ of cases, mediastinal deviation in $19.2 \%$ (tab. 2). One of the most dangerous situations is an obstruction through the valve mechanism, which causes localized hyperinflation with diaphragmatic flattening and mediastinum displacement to a healthy hemithorax with significant risks of pneumothorax (fig. 1,2).

Table 2. Pulmonary radiological changes in children with foreign body aspiration

\begin{tabular}{|l|c|c|c|}
\hline \multicolumn{1}{|c|}{ Chest radiography } & No of cases & $\mathbf{\%}$ & $\mathbf{9 5 \%} \mathbf{~ C l}$ \\
\hline Atelectasis & 39 & 25.0 & $18.4-32.6$ \\
\hline Mediastinal deviation & 30 & 19.2 & $13.4-26.3$ \\
\hline Localized emphysema & 75 & 48.1 & $40.0-56.2$ \\
\hline Pneumonic opacities & 120 & 76.9 & $69.5-83.3$ \\
\hline Normal aspect & 13 & 8.3 & $4.5-13.8$ \\
\hline
\end{tabular}
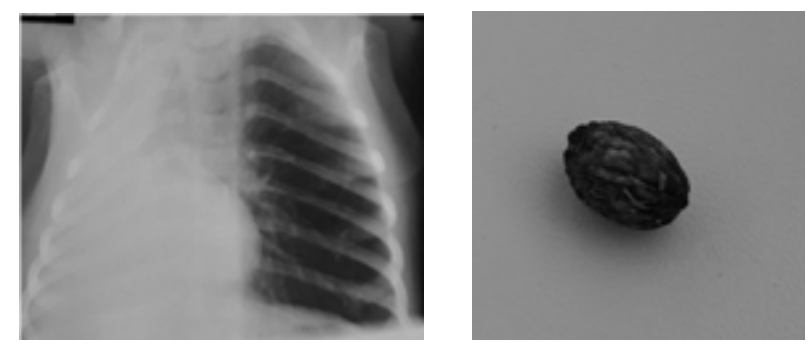

Fig. 1. Valve mechanism with left lung hyperinflation, significant deviation of the mediastinum to the right, and flattening of the left hemidiaphragm in olive kernel aspiration, located in the left main bronchus
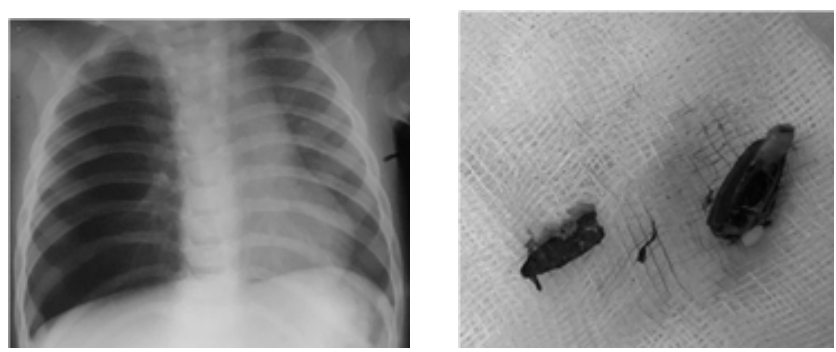

Fig. 2. Valve mechanism with right lung hyperinflation and mediastinum deviation to the left after sunflower seed aspiration, positioned in the right main bronchus

In the study the following foreign bodies were localized in the respiratory tree: trachea -2 cases $(1.3 \%)$, main right bronchus - 50 cases $(32.1 \%)$, left main bronchus - 34 cases 
(21.8\%), lobar/segmental bronchi - 35 cases $(22.5 \%)$, and multiple locations - 33 cases (21.2\%), as confirmed by bronchoscopic examination.

The nature of foreign bodies was very diverse, the organic ones predominating - 96.8\% (fig. 3). Foreign body was removed during the first bronchoscopy in $94.87 \%$ of patients. The remaining foreign body fragments were removed from the bronchial tree in $14.74 \%$ of cases at the endoscopic reassessment after 3-5 days. The most common complications were infectious, these patients subsequently requiring antibiotic therapy.

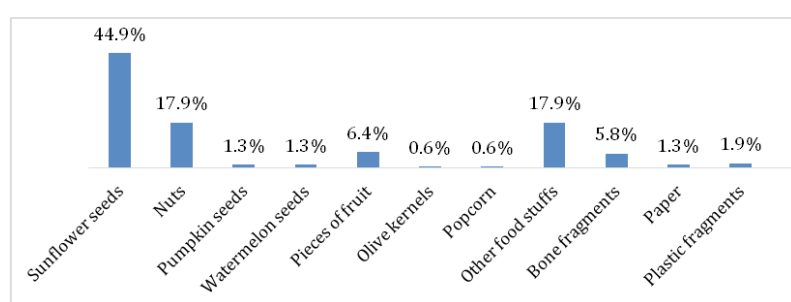

Fig. 3. The etiological structure of foreign bodies extracted from the bronchial tree in children

\section{Discussion}

The 156 cases reported over ten years indicate that this occurrence is relatively common among children. The maximum incidence aligned to information in the literature, which stated that approximately $80 \%$ of pediatric episodes of FBA occur in children under the age of three, with a maximum incidence between the ages of one and two years [17-21]. There is a variety of reasons that support aspiration, most of which are tied to this age, as youngsters are especially vulnerable at this period due to increased activity and interest, as well as reduced parental monitoring as they grow up. Additionally, young children have a high level of activity and distraction while eating and tend to explore the world with their mouths while playing. Anatomical and morphological characteristics are also important. The lack of molars increases the danger of foreign body aspiration. Because airway resistance is inversely related to the crosssectional radius, children's airways have a stronger proclivity to impede airflow, even though small foreign bodies [6].

The gender difference was statistically insignificant, with boys having a little predominance. However, studies from the literature reveal a net frequency in males of almost 2:1 $[18,20,21]$. Tomaske M. et al. on a group of 370 children with an aspiration of foreign bodies found 242 cases (65.4\%) in males [22]. Another recent study showed that $67.9 \%$ of the 316 children with foreign bodies in their airways were boys [19]. In a larger group analysis (1027 patients with foreign body aspiration), 626 boys and 401 girls were found to have them $[23,24]$.

When patients present to the emergency department with a clear history of foreign body aspiration, the diagnosis is usually clear. Difficult situations are represented by those cases in which the moment of aspiration goes unnoticed by parents or supervisors, and the child begins to develop complications (usually trenant pneumonia). When present in the anamnesis, penetration syndrome is highly suggestive of a diagnosis. In the studied group it was found in 103 cases $(66.0 \%)$. Other authors report this syndrome in $77 \%$ of cases [25]. However, it is not always visible, which causes a delay in diagnosis. Cough, dyspnea, and wheezing were the most prevalent symptoms among the patients in the study. According to the study more than 1000 patients had cough $(84.3 \%)$, dyspnea $(13.1 \%)$, and cyanosis in $7.7 \%$ of cases [24].

When there is even the slightest suspicion of foreign bodies in the airways, a radiological scan is required. Children in the study group had indirect indicators: atelectasis, mediastinal shift, and localized emphysema. The authors of the previously referenced study discovered emphysema in 707 patients $(68.8 \%)$ and atelectasis in 137 patients $(13.3 \%)$ [24]. Normal radiological result was found in 13 patients (8.3\%). A normal chest X-ray cannot rule out the existence of a foreign body [26]. In the event of a strong, clinical, or anamnestic suspicion, it is required to repeat the radiograph and maybe do tomography, which can provide more information.

Both the study and the information in the literature show that foreign bodies are mostly found in the right bronchus. The higher frequency has anatomical explanations: the right bronchus is closer to the tracheal axis than the left bronchus, it has a larger caliber, the suction force of the right lung is greater, and the carina is to the left $[12,24]$.

The nature of the aspirated foreign body differs based on geographical area, environment, culture, and eating habits, with numerous researches indicating that biological foreign bodies are more common [27]. In the Republic of Moldova, it is also observed that the aspirations of vegetable foreign bodies predominate, namely sunflower seeds and nuts.

The main cause of late arrival in specialized services is misdiagnosis due to the multiple pathological entities with which foreign body aspiration can be confused, and extraction usually takes place after prior hospitalization in pediatric wards or after inefficient and inadequate outpatient treatments with diagnosis. The progression and prognosis of foreign body aspiration are dependent on the timing of diagnosis and therapeutic intervention, and so any suspicion based on anamnesis, clinical data, and imaging data should be followed by tracheobronchoscopic investigation.

\section{Conclusions}

1. Foreign body aspiration into the airways is rather prevalent in children, particularly those aged 1-3 years.

2. The presence of penetration syndrome, followed by cough, dyspnoea, and wheezing, is very suggestive for establishing the diagnosis.

3. At the smallest suspicion of foreign body aspiration a radiological study is required, which may be indicative of unilateral pulmonary hyperinflation or lobar/segmental atelectasis.

\section{References}

1. Naragund AI, Mudhol RS, Harugop AS, et al. Tracheo-bronchial foreign body aspiration in children: a one-year descriptive study. Indian J Otolaryngol Head Neck Surg. 2014:66(Suppl 1):180-185. doi: 10.1007/ s12070-011-0416-2.

2. Mantor PC, Tuggle DW, Tunell WP. An appropriate negative bronchoscopy rate in suspected foreign body aspiration. Am J Surg. 1989;158(6):622624. https://doi.org/10.1016/0002-9610(89)90208-0. 
3. Steen KH, Zimmermann TH. Tracheobronchial aspiration of foreign bodies in children: a study of 94 cases. Laryngoscope. 1990;100(5):525530. doi: 10.1288/00005537-199005000-00016.

4. Brkić F, Umihanić Š, Altumbabić H. Delay in diagnosis of foreign body aspiration in children. Acta Medica Saliniana. 2019;49(1):7-10. https:// doi:10.5457/ams.v49i1.482.

5. Johnson K, Linnaus M, Notrica D. Airway foreign bodies in pediatric patients: anatomic location of foreign body affects complications and outcomes. Pediatr Surg Int. 2017;33(1):59-64. doi: 10.1007/s00383-0163988-9.

6. Morris IR. Anesthesia and airway management of laryngoscopy and bronchoscopy. In: Hagberg CA, editor. Benumof's airway management. 2nd ed. Philadelphia: Mosby; 2007. p. 859-888

7. Osman NM, Korraa EE, Abd Elfattah NM. Complications and follow-up of foreign body inhalation. Egyptian J Bronchol. 2016;10(3):232-237. https://doi.org/10.4103/1687-8426.193631.

8. Barrios JE, Gutierrez C, Lluna J, et al. Bronchial foreign body: should bronchoscopy be performed in all patients with a choking crisis? Pediatr Surg Int. 1997;12(2-3):118-120. https://doi.org/10.1007/BF01349976.

9. Holliday S, Gurkowski MA. Foreign body aspiration. In: Atlee JL, editor. Complications in anesthesia. 2nd ed. Philadelphia: Saunders; 2007. p. 753-755. https://doi.org/10.1016/B978-1-4160-2215-2.50193-9.

10. Ada’s Medical Knowledge Team. Foreign Body Aspiration [Internet]. Berlin: Ada Health GmbH; (C2021 [cited 2021 Jul 12]. Available from: https:/ada.com/conditions/foreign-body-aspiration/

11. Berry F, Stemland C. Foreign body aspiration. In: Fleisher LA, Roizen MF editors. Essence of anesthesia practice. Philadelphia: Elsevier/Saunders; 2011. p. 156

12. Dehghani N, Ludemann P. Aspirated foreign bodies in children: BC Children's Hospital emergency room protocol. BC Med J. 2008;50(5):252256.

13. Douira-Khomsi W, Merghni A, Louati H, et al. Epis de graminée intrabronchiques migrateurs, un corps étranger hors du commun [Intrabronchial migratory cereal-ear, an unusual foreign body inhalation]. Arch Pédiatr. 2009;16(9):1280-1283. https://doi.org/10.1016/j. arcped.2009.06.006. French.

14. Eber E, Antón-Pacheco JL, de Blic J, et al. ERS statement: interventional bronchoscopy in children. Eur Respir J. 2017;50(6):1700901. doi: 10.1183/13993003.00901-2017.

15. Karakoç F, Karadağ B, Akbenlioğlu C, et al. Foreign body aspiration: what is the outcome? Pediatric Pulmonol. 2002;34(1):30-36. https://doi. org/10.1002/ppul.10094
16. Shlizerman L, Mazzawi S, Rakover Y, et al. Foreign body aspiration in children: the effects of delayed diagnosis. Am J Otolaryngol. 2010;31(5):320-324. doi: 10.1016/j.amjoto.2009.03.007.

17. Centers for Disease Control and Prevention (CDC). Nonfatal chokingrelated episodes among children - United States, 2001. MMWR Morb Mortal Wkly Rep. 2002 Oct 25;51(42):945-8.

18. Cramer N, Jabbour N, Tavarez MM, Taylor RS. Foreign body aspiration In: StatPearls [Internet]. Treasure Island (FL): StatPearls Publishing; 2021 [cited 2021 Aug 11]. Available from: https://www.ncbi.nlm.nih. gov/books/NBK531480/

19. Kalyanappagol VT, Kulkarni NH, Bidri LH. Management of tracheobronchial foreign body aspirations in paediatric age group - a 10-year retrospective analysis. Indian J Anaesthesia 2007;51(1):20-23.

20. Warshawsky ME, Talavera F, Mosenifar Z. Foreign body aspiration. In: Medscape [Internet]. New York: WebMD; (C1994-2021 [cited $2021 \mathrm{Jul}$ 13]. Available from: https://emedicine.medscape.com/article/298940overview

21. Ruiz FE. Airway foreign bodies in children. In: UpToDate [Internet]. Waltham, MA: UpToDate; 2020 [cited 2021 May 23]. Available from: http://www.uptodate.com/contents/airway-foreign-bodies-in-children

22. Tomaske M, Gerber AC, Stocker S, et al. Tracheobronchial foreign body aspiration in children - diagnostic value of symptoms and signs. Swiss Med Wkly. 2006;136(33-34):533-538.

23. Eren S, Balci AE, Dikici B, et al. Foreign body aspiration in children: experience of 1160 cases. Ann Trop Paediatr. 2003;23(1):31-37. https:// doi.org/10.1179/000349803125002959.

24. Tang LF, Xu YC, Wang YS, et al. Airway foreign body removal by flexible bronchoscopy: experience with 1027 children during 2000-2008. World J Pediatr. 2009;5(3):191-195. doi: 10.1007/s12519-009-0036-Z.

25. Ouoaba K, Diara C, Dao MO, Ouedraogo I, et al. Les corps étrangers laryngo-trachéo-bronchiques chez l'enfant au CHU Onagadogon (une analyse de 96 observations) [Laryngo-tracheo-bronchial foreign bodies in children at the University Hospital Center of Ouagadougou (analysis of 96 cases)]. Med Trop. 2002;62:611-614. French.

26. Zerella JT, Dimler M, McGill LC, Pippus KJ. Foreign body aspiration in children: value of radiography and complications of bronchoscopy. J Pediatr Surg. 1998;33(11):1651-1654. https://doi.org/10.1016/S00223468(98)90601-7.

27. Ciftci AO, Bingöl-Koloğlu M, Senocak ME, et al. Bronchoscopy for evaluation of foreign body aspiration in children. J Pediatr Surg. 2003;38(8):1170-1176. https://doi.org/10.1016/S0022-3468(03)00263-X.

\section{Authors' ORCID iDs and academic degrees}

Diana Rotaru-Cojocari, MD, PhD Applicant - https://orcid.org/0000-0001-7867-3756

Victor Rascov, MD, PhD Applicant - https://orcid.org/0000-0002-7826-866X

Rodica Selevestru, MD, PhD, Associate Professor - https://orcid.org/0000-0002-8923-3075

Svetlana Sciuca, MD, PhD, Professor - https://orcid.org/0000-0003-1091-9419

\section{Authors' contributions}

DRC designed the research, reviewed statistics and interpreted the data, drafted the manuscript; VR collected the data, did statistical analysis; RS interpreted the data, revised the manuscript critically; SS conceptualized the project and designed the research, revised the manuscript critically.

\section{Funding}

This study was supported by Nicolae Testemitanu State University of Medicine and Pharmacy. The trial was the authors' initiative. The authors are independent and take responsibility for the integrity of the data and accuracy of the data analysis.

\section{Ethics approval and consent to participate}

The research project was approved by the Research Ethics Committee of Nicolae Testemitanu State University of Medicine and Pharmacy (Protocol No 41 of 25.05.2021)

\section{Conflict of Interests}

No competing interests were disclosed. 